\title{
Two-step procedure for trace element analysis in seafood via calibration-free laser-induced breakdown spectroscopy
}

\author{
Chia-Ting Chen ${ }^{\mathrm{a}}$, Daniela Banaru ${ }^{\mathrm{a}}$, Thierry Sarnet ${ }^{\mathrm{b}}$, Jörg Hermann ${ }^{\mathrm{b}, *}$ \\ ${ }^{a}$ Aix-Marseille University, CNRS/INSU, Toulon University, IRD, Mediterranean Institute of Oceanography, 13009 Marseille, France \\ ${ }^{b}$ Aix-Marseille University, CNRS, LP3, 13009 Marseille, France
}

\begin{abstract}
Elemental analyses via calibration-free laser-induced breakdown spectroscopy (LIBS) usually suffer low sensitivity due to continuum emission generated by the plasma in local thermodynamic equilibrium. Here we propose a two-step measurement procedure that enables improved sensitivity of calibration-free LIBS. The method consists of recording two emission spectra with different delays between the laser pulse and the detector gate. The short delay is used to probe the plasma in conditions of full local thermodynamic equilibrium in order to measure major and minor element concentrations. To evaluate the concentrations of trace elements, a second measurement with improved limits of detection is performed with a larger gate delay. In that condition, the partial equilibrium state of the plasma enables the quantification of minor and trace elements. Demonstrated via analyses of lyophilized seafood samples, the presented method is suitable for all types of food, and more general for organic materials and all materials that include elements such as carbon, oxygen, nitrogen and hydrogen, for which the equilibrium state is hardly achieved.
\end{abstract}

Keywords: LIBS; calibration-free; food analysis; seafood; trace element analysis;

\section{Introduction}

Compositional measurements of food are among the most challenging tasks in the field of analytical chemistry. Environmental pollution of industrial, urban and agricultural origins and the increasing demand of healthy alimentation generated an exponentially growing interest for quality control of aliments [1, 2, 3]. The well-established commonly-used techniques for elemental analysis of food such as atomic absorption spectroscopy (AAS), inductively coupled plasma mass spectrometry (ICP-MS) or inductively coupled plasma atomic emission spectrometry (ICP-AES) being costly and time-expensive, their use for large-scale analyses in monitoring and quality control is limited.

Laser-induced breakdown spectroscopy (LIBS) stimulated strongly growing interest in the past years due to the capability of performing rapid multielemental analyses without or with minimized sample preparation $[4,5]$. Thus, the analytical capabilities of LIBS were investigated for various types of food such as wheat $[6,7]$, cereals $[8,9]$, vegetables $[10,11]$, milk powder [12, 13], and oil [14]. The presented approaches

*Corresponding author: hermann@lp3.univ-mrs.fr that aim to quantify the elemental composition can be divided into three categories: (i) measurements based on calibration with standard samples; (ii) chemometric methods; (iii) calibration-free measurements. Due to the complex composition and the heterogeneity of the organic materials, measurements based on calibration are affected by large measurement errors. The difficulties of calibrated measurements are amplified by matrix effects that are particularly severe due to the high variability of the laser energy coupling towards the heterogeneous organic matter [3]. Chemometric methods have been successfully applied to classify food samples according to their elemental ratio $[15,16]$. However, their use for quantitative measurements without any calibration with standards is not possible. Calibration-free approaches have been applied mostly to quantify mineral elements in food or food supplements [17, 18, 19]. Based on the assumption of local thermodynamic equilibrium (LTE), the main difficulty of these techniques comes from the requirement of model validity [20, 21]. Indeed, LTE is only established in plasmas of sufficiently large electron density [22], and continuum emission associated to free charges in the plasma limits the signal-to-noise ratio of the explored atomic or molecular emission [23, 24]. Consequently, calibration-free 
laser-induced breakdown spectroscopy (CF-LIBS) was mostly applied to measure elements with sufficiently high concentration values. In addition, the application of CF-LIBS to organic materials encounters a supplementary difficulty that arises from matrix elements $\mathrm{C}$, $\mathrm{H}, \mathrm{O}$ and $\mathrm{N}$. According to the atomic structures including large energy gaps between the electronic excitation levels, equilibrium Boltzmann distributions are hardly achieved for these elements [25, 26]. The matrix elements are therefore generally ignored and CF-LIBS measurements are limited to a partial analysis of the elemental composition of food [17, 12, 18, 11, 19].

In the present paper, we present a method that improves the sensitivity of CF-LIBS, enabling thus the quantification of the full elemental composition. The two-step procedure is based on the recording of two spectra with different delays after the exciting laser pulse. The early measurement, performed at a time when the plasma is in full LTE, serves to measure the major and minor element fractions. The late measurement is performed in conditions of partial LTE to quantify minor and trace elements. Here, due to the reduced electron density, the elements of the organic matrix are out of equilibrium whereas the other atoms still have Boltzmann equilibrium distributions. The continuum radiation being lowered as a consequence of the reduced collision rates of charged particles, the signal-to-noise ratio is improved. To further improve the signal-to-noise ratio, a large gate width is applied. The variation of plasma properties during the time of observation are taken into account by dividing the gate width into two time-intervals and by calculating the spectrum through the combination of two spectra, computed for the two successive thermodynamic states.

The demonstration is given for three types of most commonly consumed Mediterranean seafood species, sampled in the bay of Marseille. This bay is highly impacted by historical and present urban and industrial pollution [27, 28, 29, 30, 31].

\section{Experiment}

The experiments were carried out in experimental conditions that were previously found to enable accurate compositional analysis via calibration-free LIBS [32]. Thus, ultraviolet $(266 \mathrm{~nm})$ laser pulses of $4 \mathrm{~ns}$ duration and $6 \mathrm{~mJ}$ pulse energy were focused onto the lyophilized seafood samples with a lens of $150 \mathrm{~mm}$ focal length. The samples were placed on a motorized sample holder in a vacuum chamber that was filled with argon at $5 \times 10^{4} \mathrm{~Pa}$ pressure during the experiments (see Fig. 1).

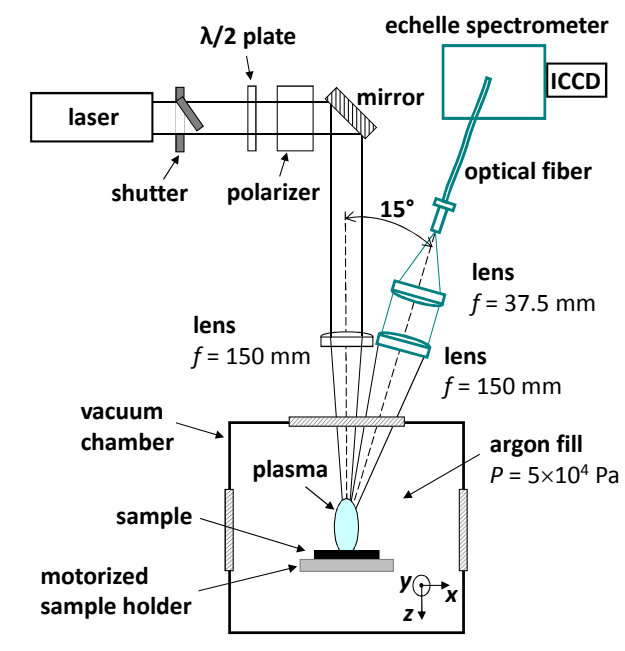

Figure 1: Schematic view of the experiment.

The LIBS spectra were recorded by imaging the plasma with two lenses of 150 and $35 \mathrm{~mm}$ focal lengths onto the entrance of an optical fiber of $600 \mu \mathrm{m}$ core diameter. According to the image magnification 1:5, the entire plasma volume was observed. The fiber was coupled to an echelle spectrometer of $0.4 \mathrm{~m}$ focal length (LTB, model Aryelle Butterfly) equipped with an intensified charge-coupled device (ICCD) matrix detector. The spectral resolution of the apparatus was measured as a function of wavelength using a low-pressure argonmercury lamp. An intensity calibration of the spectroscopic apparatus was performed in the visible and UV spectral ranges using calibrated tungsten and deuterium lamps, respectively.

The present measurements being performed with an optical system of low efficiency, the spectra were recorded by averaging over a large number of ablation events. Thus, 3 laser pulses were applied on 400 different irradiation sites, separated by a distance of $150 \mu \mathrm{m}$. We stress that the recording time can be reduced by more than two orders of magnitude if an optical system of high efficiency is used.

The three considered samples, common cuttlefish (Sepia officinalis Linnaeus, 1758), common octopus (Octopus vulgaris Cuvier, 1797), and European pilchard (Sardina pilchardus (Walbaum, 1792)) are labelled sepia, octopus, and sardine, respectively. For each sample, the white muscle was extracted and kept frozen before being freeze-dried. 


\section{Description of measurement procedure}

\subsection{Two-step measurement procedure}

The two-step LIBS measurements were performed by recording spectra with two different delays of the detector gate with respect to the laser pulse (see Fig. 2). The early recording is performed at a time when the electron density is large enough to ensure full LTE, enabling thus to measure the fractions of major and minor elements. For this measurement, the gate width $\Delta t_{\text {gate }}$ is set small enough so that the variations of temperature $T$ and electron density $n_{e}$ during the time of observation are small compared to their absolute values. We have shown in previous studies that a gate width $\Delta t_{\text {gate }} \leq t_{\text {gate }} / 2$ is a reasonable compromise between model validity and signal-to-noise ratio [25, 26]. The late measurement is performed with a large gate width to reach highest signal-to noise ratio required for trace element detection. At that time, the plasma in partial equilibrium enables the quantification of minor and trace elements. As the variations of $T$ and $n_{e}$ are not negligible during the observation time, they are taken into account in the calculation of the plasma emission spectrum (see Section 4.4). The attributes of both measurements are summarized in Table 1.

The values of gate delay and gate width applied in both measurements were chosen based on the knowledge acquired in previous investigations. These studies evidenced that accurate compositional measurements of oxygen-containing materials such as glass and sapphire are possible only for recording times $<1 \mu \mathrm{s}$, when the electron density is large enough to ensure collisional equilibrium $[25,26]$. For later recording times, the atom with the largest energy gap between excited states,

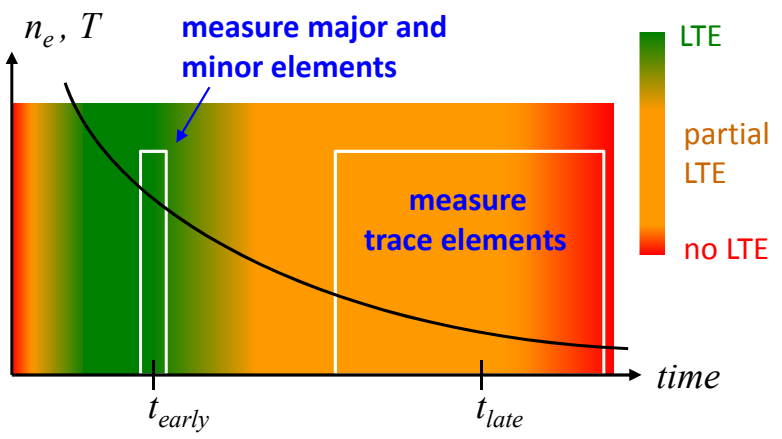

Figure 2: Time scheme of two-step procedure: two spectra are recorded at different times. The early recording in conditions of full LTE serves to quantify major and minor elements. The late recording in conditions of partial LTE serves to measure minor and trace elements. The color scale from green to red indicates the degree of equilibrium.
Table 1: Early and late recordings of the two-step procedure: measurement time $t=t_{\text {gate }}+\Delta t_{\text {gate }} / 2 \pm \Delta t_{\text {gate }} / 2$, gate delay $t_{\text {gate }}$, gate width $\Delta t_{\text {gate }}$, plasma equilibrium state during measurement, signal-tonoise ratio, and measured elements.

\begin{tabular}{ccc}
\hline measurement & early & late \\
\hline$t(\mu \mathrm{s})$ & $0.35 \pm 0.05$ & $4.5 \pm 2.5$ \\
$t_{\text {gate }}(\mu \mathrm{s})$ & 0.30 & 2.0 \\
$\Delta t_{\text {gate }}(\mu \mathrm{s})$ & 0.10 & 5.0 \\
plasma state & full LTE & partial LTE \\
S/N ratio & low & high \\
measured elements & major, minor & minor, trace
\end{tabular}

namely oxygen, was shown to run out of equilibrium, leading to an overestimation of the oxygen fraction. We consequently expect for the here chosen early measurement time of $0.35 \mu$ s Boltzmann equilibrium distributions for all elements of the organic samples. The choice of the late measurement delay is founded on calibration-free analysis under equal experimental conditions, showing that accurate compositional analyses of metals can be performed with measurement times as late as several microseconds [26].

We stress that, even when the equilibrium conditions are not fully satisfied, the resulting measurement errors of the minor and trace elements are expected to be small compared to statistical variations due to the heterogeneity of the samples [33].

For heterogeneous samples, the measured composition naturally depends on the probed volume. Weak fluctuations of element fractions with the probed volume are expected for major elements whereas much larger fluctuations are expected for minor and trace elements. The measured composition is thus representative of the volume probed during the acquisition of the late spectrum used for the trace element analysis.

\subsection{Calculation algorithm}

The LIBS measurement algorithm is presented schematically in Fig. 3. It consists of two iteration loops. In the principal loop (a), electron density, temperature, elemental fractions, and plasma diameter are successively measured. Each measurement is performed by executing the loop (b) in which the computed spectrum is compared to the measured one while the parameter to be measured is varied. The best agreement obtained from the $\chi^{2}$-test determines the measurement result. The LIBS measurement is successfully terminated when the changes of all measured parameters are small compared to their absolute values. 


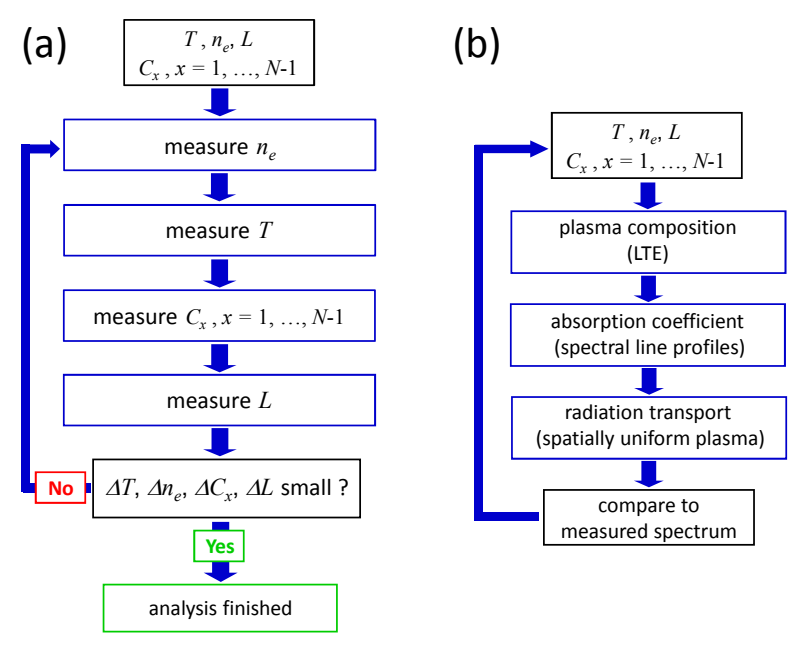

Figure 3: LIBS measurement algorithm: (a) principal iteration loop with successive measurements of electron density, temperature, elemental fractions, and plasma diameter. Each measurment is performed using the calculation loop (b) by varying only the parameter to be measured.

\subsection{Calculation of spectral radiance}

According to previous work [32], the plasma produced under the present experimental conditions appears spatially uniform. Assuming local thermodynamic equilibrium, the spectral radiance generated by the plasma can be computed using [34]

$$
B_{\lambda}=U_{\lambda}\left(1-e^{-\alpha(\lambda) L}\right),
$$

where $U_{\lambda}$ is the blackbody spectral radiance, $\alpha(\lambda)$ the wavelength-dependent absorption coefficient, and $L$ the plasma size along the line of sight. Neglecting the contributions of bremsstrahlung and recombination, only transitions between bound levels are considered and the absorption coefficient writes [22]

$$
\alpha_{\text {line }}(\lambda)=\pi r_{0} \lambda^{2} f_{l u} n_{l} P(\lambda)\left(1-e^{-h c / \lambda k T}\right) .
$$

Here $r_{0}$ is the classical electron radius, $h$ is the Planck constant, $c$ is the vacuum light velocity, $k$ is the Boltzmann constant, $f_{l u}$ and $n_{l}$ are the absorption oscillator strength and the lower level population number density of the transition, respectively. The normalized line profile $P(\lambda)$ is calculated considering Doppler and Stark broadening that are the dominant mechanisms of spectral line broadening in strongly ionized laser-produced plasmas [35]. Depending on the relative values of Doppler and Stark widths, the line shapes are described by Gaussian, Lorentzian or Voigt profiles. The Doppler width is calculated according to plasma temperature and atomic mass. The Stark width of each spectral line is obtained using Stark broadening parameters $w$ and assuming a linear dependence of the Stark width with the electron density $[36,37]$.

\subsection{Calculation of plasma chemical composition}

The chemical composition of the plasma in local thermodynamic equilibrium is computed using the calculation scheme presented in previous work $[38,25]$. The calculations include the formation of diatomic species via chemical reactions. Here, we only consider diatomics of sufficiently large dissociation energy so that the corresponding number densities have an influence on the plasma chemical composition.

For a plasma in LTE composed of $N$ elements, the composition depends on $N+1$ parameters: the temperature and the atomic number densities of the $N$ elements. In the temperature range of interest the formation of polyatomic molecules can be neglected and the atomic number density of an element $A$ is given by

$$
n_{A}=\sum_{z=0}^{z_{\max }} n_{A}^{z}+2 \sum_{z=0}^{1} n_{A_{2}}^{z}+\sum_{B \neq A} \sum_{z=0}^{1} n_{A B}^{z},
$$

Here, $n_{A}^{z}$ and $n_{A_{2}}^{z}$ are the number densities of atomic and homonuclear diatomic species of charge $z$, respectively, $n_{A B}^{z}$ are the number densities of heteronuclear diatomic species of charge $z$ formed by chemical reactions with the element $B$. The sum includes all species of significant abundance up to the maximum charge $z_{\text {max }}$. The number densities of diatomic molecules formed by chemical reactions between the elements $A$ and $B$ were obtained from the Guldberg-Waage law of mass action for chemical equilibrium [39]

$$
\frac{n_{A}^{0} n_{B}^{0}}{n_{A B}^{0}}=\frac{(2 \pi \mu k T)^{3 / 2}}{h^{3}} \frac{Q_{A}^{0} Q_{B}^{0}}{Q_{A B}^{0}} e^{-D_{0} / k T},
$$

where $n_{A}^{0}$ and $n_{B}^{0}$ are the number densities of neutral atoms of elements $A$ and $B$, respectively, $n_{A B}^{0}$ is the density of diatomic molecules formed by chemical reactions between both elements. $Q_{A}^{0}, Q_{B}^{0}$ and $Q_{A B}^{0}$ are the corresponding partition functions. $D_{0}$ is the dissociation energy of the diatomic molecule $A B$ in the ground state, $\mu=m_{A} m_{B} /\left(m_{A}+m_{B}\right)$ is the reduced mass, $m_{A}$ and $m_{B}$ are the atomic masses of elements $\mathrm{A}$ and $\mathrm{B}$. Thus, for given values of temperature and atomic number densities $n_{A}$ of the $N$ elements, the number densities of all plasma species can be calculated by numerically solving the equations that govern the LTE plasma [38]. Once the number densities of all species are calculated, the electron density is obtained from the equation of charge 


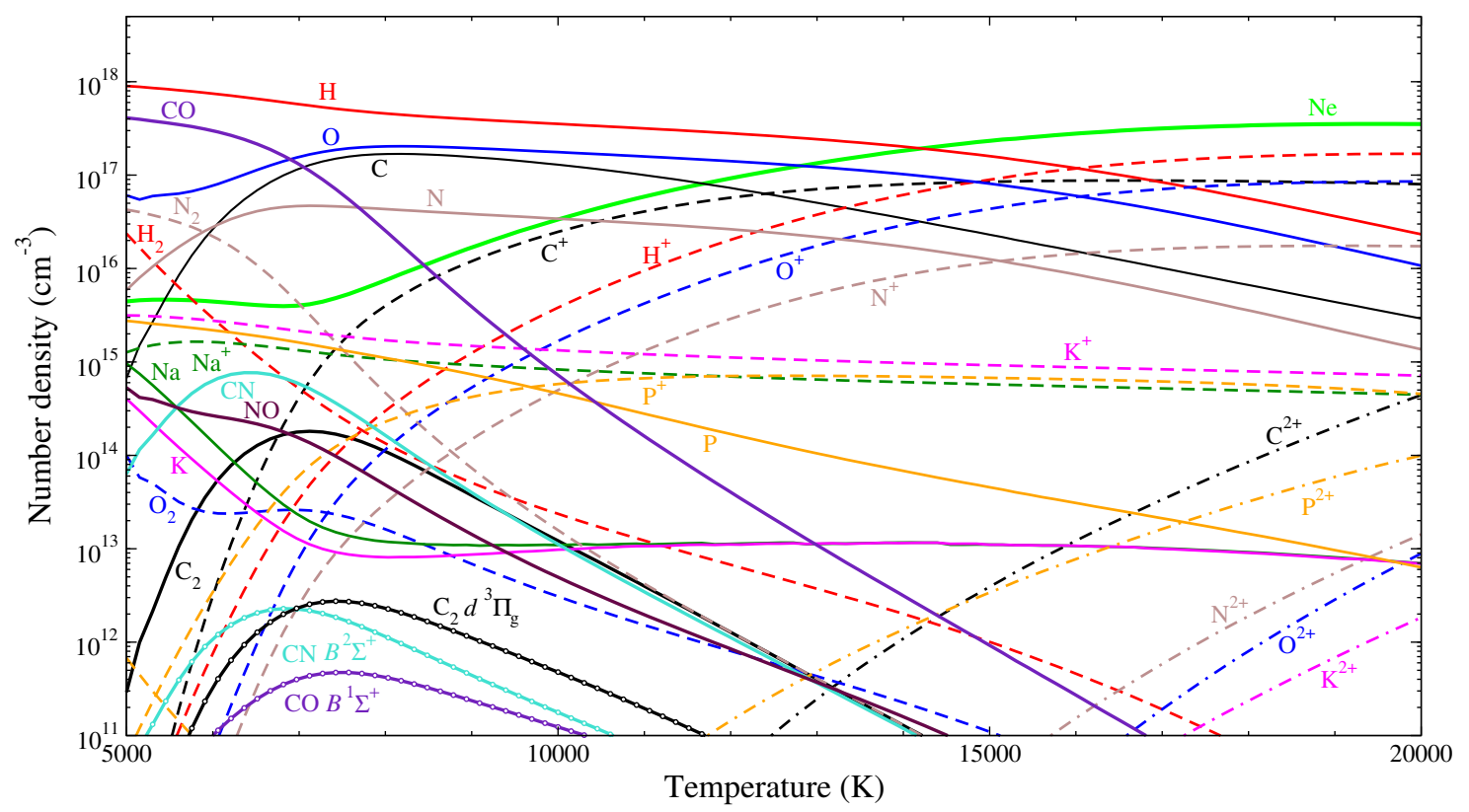

Figure 4: Number densities of plasma species versus temperature computed for a plasma in LTE at atmospheric pressure with the elemental composition of the sepia sample (see Table 3). For simplification, only elements with mass fractions $>0.1 \%$ are considered. The upper level population number densities of the most prominent molecular systems are also displayed.

neutrality by summing the densities of all charged particles,

$$
n_{e}=\sum_{A} \sum_{z=1}^{z_{\max }} z n_{A}^{z}
$$

For practical applications, the atomic number densities $n_{A}$ [see Eq. (3)] of the $N$ elements can be replaced by an equivalent set of input parameters: the electron density and the mass fractions of $N-1$ elements. The mass fraction of element $A$ is related to the atomic number density via

$$
C_{A}=n_{A} m_{A} / \rho_{t o t},
$$

where $\rho_{t o t}=\sum_{A} n_{A} m_{A}$ is the total mass density of the plasma.

In Fig. 4, the number densities of plasma species are presented as functions of temperature for the elemental composition of the sepia sample (see Table 3 ). The calculations were performed by setting the kinetic pressure of the plasma

$$
P=\left[n_{e}+\sum_{A} \sum_{z=0}^{z_{\max }}\left(n_{A}^{z}+n_{A_{2}}^{z}+\sum_{B \neq A} n_{A B}^{z}\right)\right] k T-\Delta P
$$

to atmospheric pressure. Here, $\Delta P$ is the Debye pressure correction that accounts for the potential energy of charged species in the plasma.
The atmospheric pressure is here an arbitrary choice that corresponds to a time between the early and late measurements [40]. According to the strong dependence of the chemical equilibrium on temperature and the weaker dependence on pressure, the temperature-dependence of plasma species shown in Fig. 4 represents qualitatively both measurements. We stress that the assumption of atmospheric pressure is only applied here for illustration. In the analysis of the measured spectra, the appropriate total density or pressure is considered via the evaluation of electron density, temperature, and relative elemental fractions in the LIBS measurement algorithm (see Fig. 3).

In addition to the number densities of atomic and molcular species, the upper level population number densities of the most prominent molcular systems are displayed in Fig. 4. It is shown that diatomic species formed by chemical reactions present a large contribution to the plasma composition. In particular, most carbon and oxygen atoms recombine to $\mathrm{CO}$ radicals for $T<7000 \mathrm{~K}$. Other diatomics have much lower number densities. Among them, $\mathrm{CN}$ and $\mathrm{C}_{2}$ reach their maximum number densities for $T=6500$ and $7500 \mathrm{~K}$, respectively. At lower temperature, most carbon atoms recombine with oxygen due to the large dissociation energy of CO [41]. Although the number density of CO radicals is orders of magnitude larger than the number 


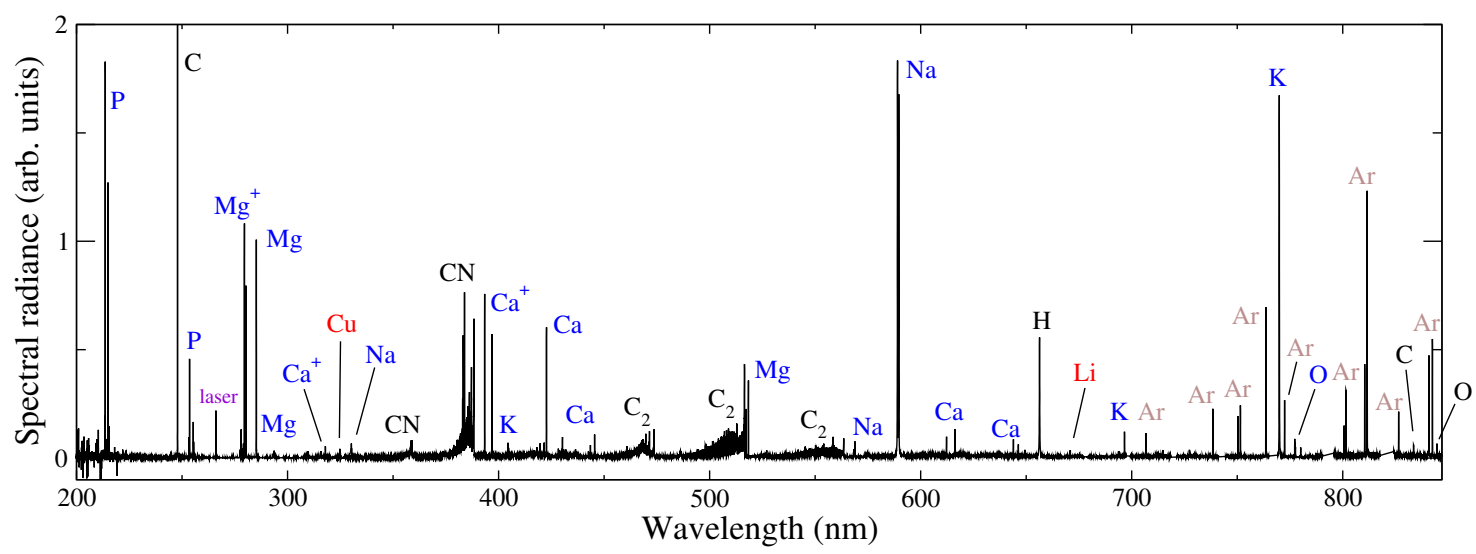

Figure 5: Spectrum recorded for sepia with a delay of $2 \mu$ s between the laser pulse and the detector gate. The gate width was set to $5 \mu$ s.

densities of $\mathrm{C}_{2}$ and $\mathrm{CN}$, the upper level number density of the $\mathrm{CO} B{ }^{1} \Sigma^{+}$state is significantly lower than the number densities of the $\mathrm{CN} B^{2} \Sigma^{+}$and $\mathrm{C}_{2} d^{3} \Pi_{g}$ states. Attributed to its high excitation energy, the low population number density of the $\mathrm{CO} B{ }^{1} \Sigma^{+}$state illustrates why molecular bands from $\mathrm{CN}$ and $\mathrm{C}_{2}$ have typically much larger emission intensity compared to $\mathrm{CO}$ emission [42].

For $T<7000 \mathrm{~K}$, the plasma electrons mostly originate from metal atoms according to their low ionization energies. At larger temperatures, ionization of carbon, hydrogen and oxygen delivers most of the electrons. Doubly charged ions play a minor role in the temperature range of interest.

\section{Results and discussion}

\subsection{Selection of analytical transitions}

A spectrum recorded for sepia is displayed in Fig. 5. The scale of the radiance axis is adjusted to the most intense lines of phosphorus, sodium and potassium, causing the dominating C I $247.85 \mathrm{~nm}$ line to be cut. The large abundance of carbon is illustrated by the observation of other atomic transitions in the infrared spectral range and several vibrational sequences of the $C_{2}$ Swan system. Except nitrogen, all matrix elements are characterized by atomic transitions of significant emission intensity. For most mineral elements, several spectral lines are observable and the most valuable analytical lines are chosen according to the following criteria: (i) the signal-to-noise ratio is large enough; (ii) no interference with lines from other elements; (iii) self-absorption is small; (iv) reliable spectroscopic data are available.
Table 2: Wavelength $\lambda$, transition probability $A_{u l}$, energy $E$ of upper (index $u$ ) and lower (index $l$ ) excitation levels of selected spectral lines. The use in temperature-, electron density-, early- and late- concentration measurements is indicated in the last columns.

\begin{tabular}{|c|c|c|c|c|c|c|c|c|}
\hline & $\begin{array}{c}\lambda \\
(\mathrm{nm})\end{array}$ & $\begin{array}{r}A_{u l} \\
(\mu \mathrm{s})\end{array}$ & $\begin{array}{r}E_{l} \\
(\mathrm{eV})\end{array}$ & $\begin{array}{r}E_{u} \\
(\mathrm{eV})\end{array}$ & $\begin{array}{c}T \\
\text { meas }\end{array}$ & $\begin{array}{c}n_{e} \\
\text { meas }\end{array}$ & $\begin{array}{l}\text { early } \\
\text { meas }\end{array}$ & $\begin{array}{l}\text { late } \\
\text { meas }\end{array}$ \\
\hline $\mathrm{Mg} \mathrm{I}$ & 202.58 & 61.2 & 0.00 & 6.12 & $\mathrm{x}$ & - & - & $\mathrm{x}$ \\
\hline $\mathrm{Zn} \mathrm{I}$ & 213.85 & 703.9 & 0.00 & 5.80 & - & - & - & $\mathrm{x}$ \\
\hline $\mathrm{Cd} \mathrm{I}$ & 228.80 & 920.1 & 0.00 & 5.42 & - & - & - & $\mathrm{x}$ \\
\hline As I & 228.81 & 382.7 & 1.35 & 6.77 & - & - & - & $\mathrm{x}$ \\
\hline As I & 234.98 & 629 & 1.31 & 6.59 & - & - & - & $\mathrm{x}$ \\
\hline B I & 249.68 & 84 & 0.00 & 4.96 & - & - & - & $\mathrm{x}$ \\
\hline B I & 249.77 & 168 & 0.00 & 4.96 & - & - & - & $\mathrm{x}$ \\
\hline P I & 255.32 & 71 & 2.32 & 7.18 & - & - & $\mathrm{x}$ & $\mathrm{x}$ \\
\hline P I & 255.49 & 30 & 2.32 & 7.18 & - & - & $\mathrm{x}$ & $\mathrm{x}$ \\
\hline Mg I & 277.66 & 132 & 2.71 & 7.18 & $\mathrm{x}$ & - & - & $\mathrm{x}$ \\
\hline $\mathrm{Mg} \mathrm{I}$ & 277.98 & 409 & 2.72 & 7.18 & $\mathrm{x}$ & - & $\mathrm{x}$ & - \\
\hline Mg I & 278.14 & 542 & 2.71 & 7.17 & $\mathrm{x}$ & - & $\mathrm{x}$ & - \\
\hline Mg I & 278.29 & 214 & 2.72 & 7.17 & $\mathrm{x}$ & - & - & $\mathrm{x}$ \\
\hline Mg II & 279.07 & 401 & 4.42 & 8.86 & $\mathrm{x}$ & - & $\mathrm{x}$ & - \\
\hline Mg II & 279.55 & 260 & 0.00 & 4.43 & $\mathrm{x}$ & - & - & $\mathrm{x}$ \\
\hline Mg II & 279.79 & 479 & 4.43 & 8.86 & $\mathrm{x}$ & - & $\mathrm{x}$ & - \\
\hline Mg II & 280.27 & 257 & 0.00 & 4.43 & $\mathrm{x}$ & - & - & $\mathrm{x}$ \\
\hline Mg I & 285.21 & 491 & 0.00 & 4.35 & $\mathrm{x}$ & - & $\mathrm{x}$ & - \\
\hline Si I & 288.16 & 217 & 0.78 & 5.08 & - & - & - & $\mathrm{x}$ \\
\hline $\mathrm{Fe} \mathrm{I}$ & 302.06 & 75.9 & 0.00 & 4.10 & - & - & - & $\mathrm{x}$ \\
\hline Mg I & 309.68 & 49.6 & 2.72 & 6.72 & $\mathrm{x}$ & - & - & $\mathrm{x}$ \\
\hline $\mathrm{Cu} \mathrm{I}$ & 327.39 & 137.6 & 0.00 & 3.79 & - & - & - & $\mathrm{x}$ \\
\hline $\mathrm{Na} \mathrm{I}$ & 330.23 & 2.75 & 0.00 & 3.75 & - & - & - & $\mathrm{x}$ \\
\hline Mg I & 382.93 & 89.9 & 2.71 & 5.95 & $\mathrm{x}$ & - & $\mathrm{x}$ & $\mathrm{x}$ \\
\hline $\mathrm{Mg} \mathrm{I}$ & 383.23 & 121 & 2.71 & 5.95 & $\mathrm{x}$ & - & $\mathrm{x}$ & $\mathrm{x}$ \\
\hline Mg I & 383.82 & 161 & 2.71 & 5.95 & $\mathrm{x}$ & - & $\mathrm{x}$ & $\mathrm{x}$ \\
\hline $\mathrm{CN}$ & Violet & 1.66 & 0.00 & 3.19 & - & - & $\mathrm{x}$ & - \\
\hline K I & 404.41 & 1.16 & 0.00 & 3.06 & - & - & - & $\mathrm{x}$ \\
\hline K I & 404.72 & 1.07 & 0.00 & 3.06 & - & - & - & $\mathrm{x}$ \\
\hline Sr II & 407.77 & 141 & 0.00 & 3.04 & - & - & - & $\mathrm{x}$ \\
\hline $\mathrm{Ca} I$ & 445.47 & 87 & 1.90 & 4.68 & - & - & - & $\mathrm{x}$ \\
\hline $\mathrm{C}_{2}$ & Swan & 8.20 & 0.09 & 2.48 & - & - & $\mathrm{x}$ & $\mathrm{x}$ \\
\hline H I & 656.2 & ? & 10.20 & 12.09 & - & $\mathrm{x}$ & $\mathrm{x}$ & $\mathrm{x}$ \\
\hline Li I & 670.77 & 36.89 & 0.00 & 1.85 & - & - & - & $\mathrm{x}$ \\
\hline $\mathrm{Li} \mathrm{I}$ & 670.79 & 36.89 & 0.00 & 1.85 & - & - & - & $\mathrm{x}$ \\
\hline C I & 833.51 & 35.1 & 7.68 & 9.17 & - & - & $\mathrm{x}$ & $\mathrm{x}$ \\
\hline
\end{tabular}

\subsection{Time-evolution of plasma properties}

Based on the successive measurements with different recording delays, the two-step procedure requires the 


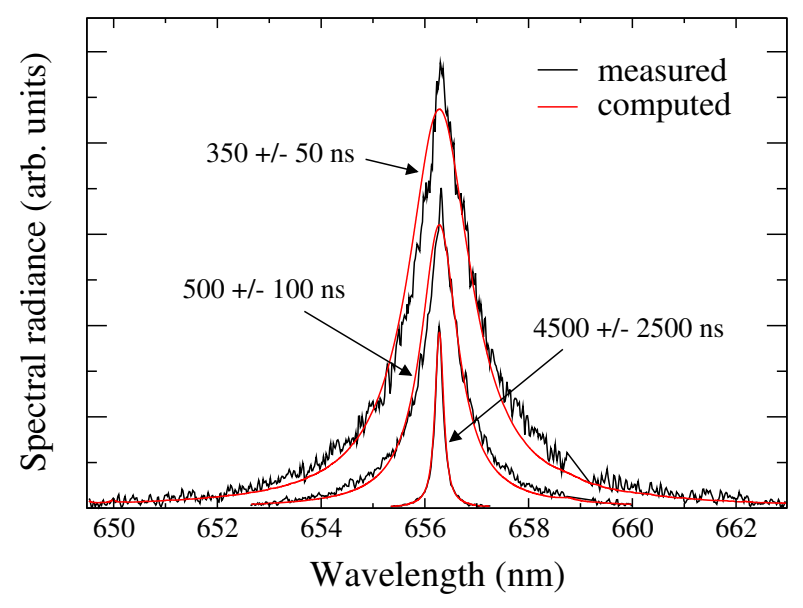

Figure 6: Measured (black line) and computed (red line) line profile of the $\mathrm{H}_{\alpha}$ transition for different observation times.

knowledge of the time-evolution of electron density and temperature. According to the task of each measurement step (see Table 1), the electron density has to be large enough to satisfy full and partial LTE conditions during the early and late measurements, respectively. In addition, the temperature has to be large enough to enable significant excitation of all analytical transitions.

The electron density was deduced from Stark broadening of the $\mathrm{H}_{\alpha}$ transition using the temperatureindependent Stark width expression $\Delta \lambda_{\text {Stark }}=f\left(n_{e}\right)$ proposed by Gigosos et al. [43]. Spectral line profiles of $\mathrm{H}_{\alpha}$ are displayed in Fig. 6 for different recording times. The measured spectra are compared to the computed spectral radiance. We remind that the calculation of the spectral line profile includes all relevant sources of line broadening (see Section 3.3).

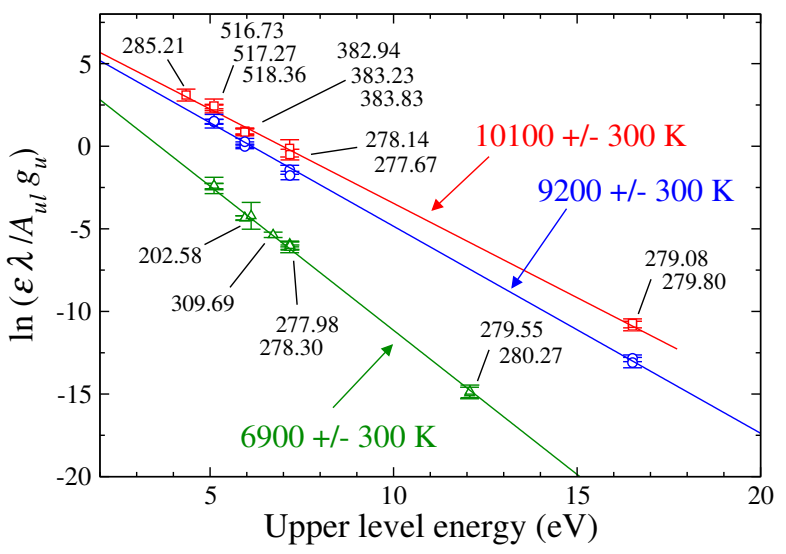

Figure 7: Saha-Boltzmann plot of $\mathrm{Mg}$ and $\mathrm{Mg}^{+}$transitions for $t=350 \mathrm{~ns}$ (squares), $500 \mathrm{~ns}$ (circles), and $4500 \mathrm{~ns}$ (triangles).

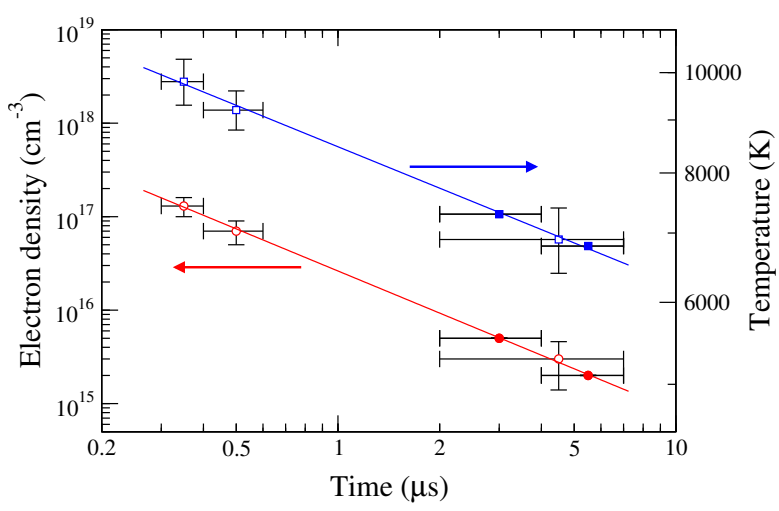

Figure 8: Electron density (circles) and temperature (squares) versus time. The vertical error bars represent the measurement uncertainties, the horizontal error bars indicate the time interval of observation (gate width). The spectrum recorded with $t=4.5 \pm 2.0 \mu$ s was compared to a computed spectrum obtained by the superposition of two spectra calculated for different $n_{e}$ - and $T$-values indicated by the filled symbols.

Accurate temperature measurements have been achieved using transitions of magnesium. Compared to the transition probabilities of other elements such as calcium or phosphorus, the $A_{u l}$-values tabulated in the NIST database [44] for magnesium have significantly higher accuracy. The measurements take benefit from the spatially uniform character of the plasma produced under the present experimental conditions. Thus, spectral line emission of atoms and ions originates from the same plasma volume and the temperature can be deduced from the simultaneous observation of neutral and ionic transitions. The temperature measurement is illustrated in Fig. 7 for different recording times. Following the criteria given in Section 4.1, the ensemble of spectral lines changes with the measurement time. For example, the $\mathrm{Mg}$ I $285.21 \mathrm{~nm}$ resonance transition is only considered in the earliest measurement, when magnesium is mostly ionized and self-absorption of the transition is moderate.

The values of electron density and temperature measured for $t=350 \pm 50 \mathrm{~ns}, 500 \pm 100 \mathrm{~ns}$, and $4.5 \pm 2.0 \mu \mathrm{s}$ are displayed in Fig. 8 on a double logarithmic scale. The temperoral evolution was approximated by functions $f(t)=A t^{B}$ represented by the continuous lines. This approximation was shown to describe closely the variations $n_{e}(t)$ and $T(t)$ in plasmas produced by laser ablation of different materials under indentical irradiation conditions [32, 40, 24]. 


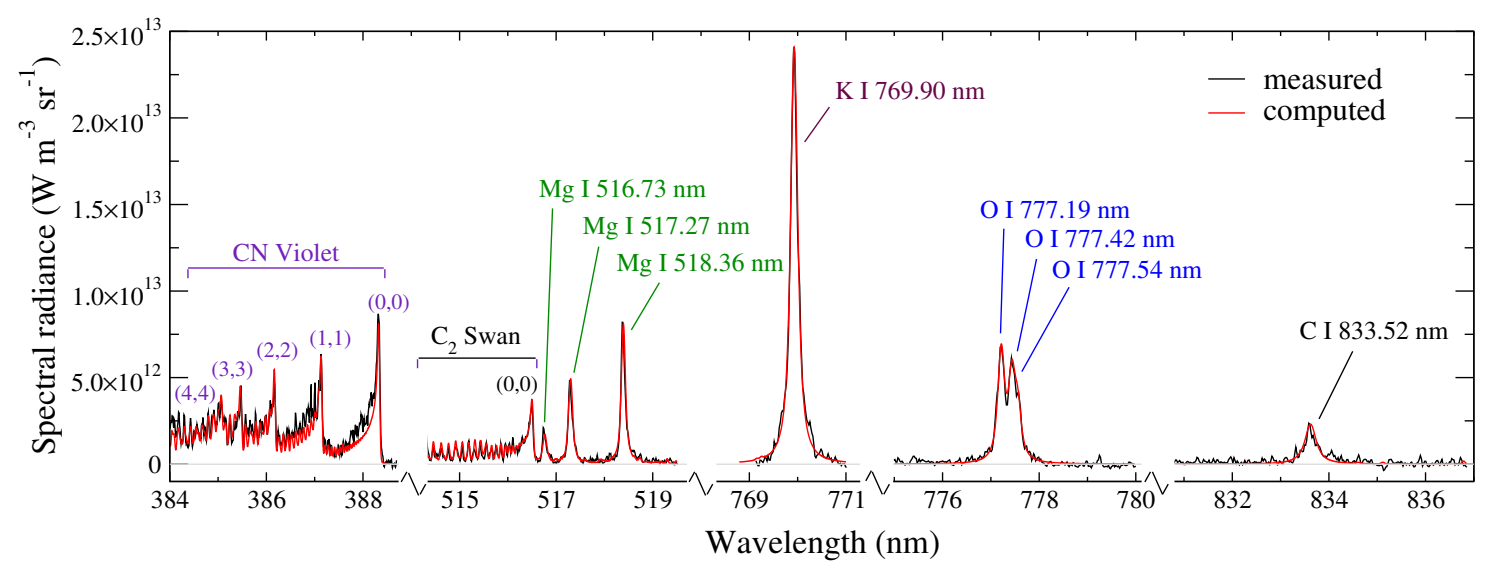

Figure 9: Molecular bands and atomic transitions emitted from major and most abundant minor elements. The spectrum recorded for sepia at $t=350 \mathrm{~ns}$ is compared to the spectral radiance computed for a plasma in local thermodynamic equilibrium (see Table 3 ).

\subsection{Measurement of major and minor elements}

The spectral ranges displayed in Fig. 9 show atomic lines and molecular bands recorded during the early measurement for the determination of major and minor elements. Due to the moderate temperature $(T<$ $10,000 \mathrm{~K}$ ), atomic transitions of nitrogen were observed with very low signal-to-noise ratio. The nitrogen content was therefore deduced from the intensity of the $\mathrm{CN}$ Violet emission bands. The amount of carbon was measurable via atomic transitions in the infrared spectral range and the prominent $\mathrm{C}_{2}$ Swan molecular emission. The good agreement between measured and computed spectra for both emission features support the validity of the LTE calculations. Indeed, the intensity ratio of atomic and molecular carbon emission depends on the temperature, on the atomic number density of carbon [see Eq. (3)], and the amount of oxygen that will reduce the available amount of carbon due to oxidation. We stress that the pressure of the laser-produced plasma at $t=350 \mathrm{~ns}$ is significantly higher than atmospheric pressure. Consequently, the atomic number densities are larger than the values considered in the calculation of the plasma composition presented in Fig. 4, and the relative amount of molecules formed by chemical reactions overcomes the amount observed for atmospheric pressure.

\subsection{Measurement of trace elements}

Due to the large gate width of the late recording, electron density and temperature undergo substantial variations during the measurement time. The spectral line intensities having nonlinear dependence on $n_{e}$ and $T$, the comparison of the measured spectrum to the spectral radiance calculated for time-invariable plasma would lead to large errors in the deduced elemental composition. For example, elements observed via transitions of relatively high excitation energy, such as phosphorus (see Table 2), are mostly excited during the early time of ob-

Table 3: Electron density $n_{e}$, temperature $T$, and plasma size $L$ used for the spectra simulation of early and late measurements. The late measurement is described by dividing it into two time-intervals, $t_{1}$ and $t_{2}$ denote the starting and ending of each interval, respectively. The mass fractions of elements deduced from both measurments are given for sepia.

\begin{tabular}{|c|c|c|c|}
\hline measurement & early & \multicolumn{2}{|c|}{ late } \\
\hline$t(\mu \mathrm{s})$ & $0.35 \pm 0.05$ & $3.0 \pm 1.0$ & $5.5 \pm 1.5$ \\
\hline$t_{1} \ldots t_{2}(\mu \mathrm{s})$ & $0.3 \ldots 0.4$ & $2 \ldots 4$ & $4 \ldots 7$ \\
\hline$n_{e}\left(\mathrm{~cm}^{-3}\right)$ & $1.3 \times 10^{17}$ & $6.2 \times 10^{15}$ & $2.0 \times 10^{15}$ \\
\hline$T(\mathrm{~K})$ & 9,800 & 7,600 & 6,800 \\
\hline$L(\mathrm{~mm})$ & 0.4 & 0.7 & 0.8 \\
\hline $\mathrm{O}(\%)$ & 50.5 & \multicolumn{2}{|c|}{-} \\
\hline $\mathrm{C}(\%)$ & 32.8 & \multicolumn{2}{|c|}{ - } \\
\hline $\mathrm{N}(\%)$ & 8.6 & \multicolumn{2}{|c|}{ - } \\
\hline $\mathrm{H}(\%)$ & 6.3 & \multicolumn{2}{|c|}{-} \\
\hline $\mathrm{K}(\%)$ & 1.0 & \multicolumn{2}{|c|}{1.0} \\
\hline $\mathrm{P}(\%)$ & 0.5 & \multicolumn{2}{|c|}{0.6} \\
\hline $\mathrm{Na}(\%)$ & 0.3 & \multicolumn{2}{|c|}{0.15} \\
\hline $\mathrm{Mg}(\mathrm{ppm})$ & - & \multicolumn{2}{|c|}{460} \\
\hline $\mathrm{Ca}(\mathrm{ppm})$ & - & \multicolumn{2}{|c|}{360} \\
\hline As (ppm) & - & \multicolumn{2}{|c|}{41} \\
\hline $\mathrm{Si}(\mathrm{ppm})$ & - & \multicolumn{2}{|c|}{5.8} \\
\hline $\mathrm{Zn}(\mathrm{ppm})$ & - & \multicolumn{2}{|c|}{5.5} \\
\hline $\mathrm{Cu}(\mathrm{ppm})$ & - & \multicolumn{2}{|c|}{4.9} \\
\hline $\mathrm{Cd}(\mathrm{ppm})$ & - & \multicolumn{2}{|c|}{1.9} \\
\hline B (ppm) & - & \multicolumn{2}{|c|}{5.8} \\
\hline $\mathrm{Li}(\mathrm{ppm})$ & - & \multicolumn{2}{|c|}{1.3} \\
\hline $\mathrm{Sr}(\mathrm{ppm})$ & - & \multicolumn{2}{|c|}{0.14} \\
\hline
\end{tabular}




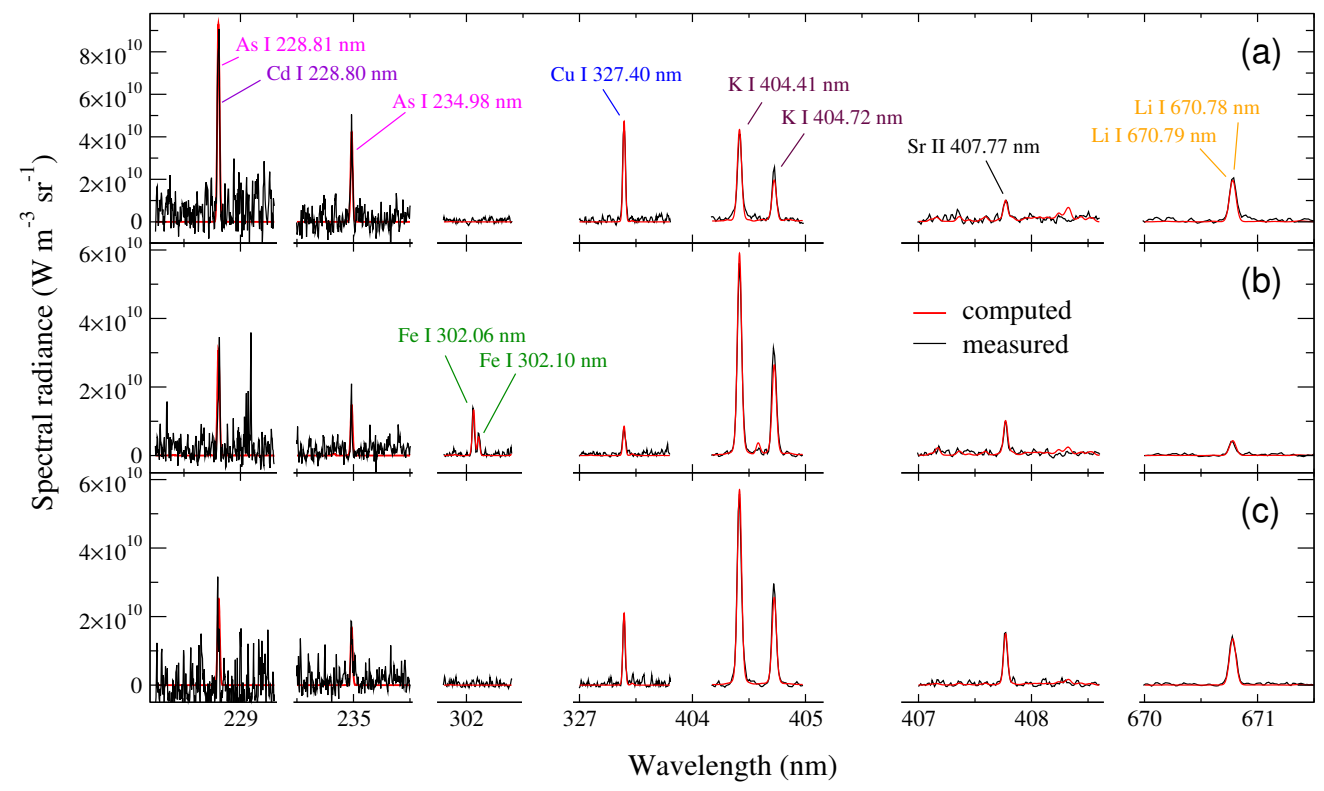

Figure 10: Transitions of minor and trace elements of spectra recorded for sepia (a), sardine (b), and octopus (c) with $t=4.5 \pm 2.5 \mu \mathrm{s}$. The measured spectra are compared to the spectral radiance computed for time-variable LTE plasma emission, considering two time-intervals of different thermodynamic state (see Table 3 ).

servation, when the temperature is large. Thus, comparing measured intensity the spectral radiance computed for a lower (average) temperature would lead to an overestimation of the elemental fraction.

To improve the accuracy of spectra simulation for the late recording, the time of observation $\Delta t_{\text {gate }}=5 \mu \mathrm{s}$ is divided into two successive time-windows of 2 and $3 \mu$ s duration. For each time-window, the plasma is characterized by taking the $n_{e^{-}}$and $T$-values (see filled symbols) deduced from $A t^{B}$-evolution indicated by the solid lines in Fig. 8. The computed spectrum is then obtained by summing the spectral radiances calculated for the two successive plasma states

$$
B_{\lambda}=\frac{\Delta t_{1}}{\Delta t_{\text {gate }}} B_{\lambda, 1}+\frac{\Delta t_{2}}{\Delta t_{\text {gate }}} B_{\lambda, 2} .
$$

The radiance values $B_{\lambda, 1}$ and $B_{\lambda, 2}$ are weighted by the duration of the corresponding time-intervals $\Delta t_{1}$ and $\Delta t_{2}$, respectively. The plasma properties used for both time-windows to describe the late measurement are listed in Table 3 together with the values used for the simulation of the early measurement.

The spectral ranges displayed in Fig. 10 show the analytical lines of several minor and trace elements observed for three different seafood species. The presence of arsenic is clearly evidenced for all samples by the observation of two transitions in the ultraviolet spectral range. The most intense line of cadmium is interfered with the As I $228.81 \mathrm{~nm}$ transition. However, a small amount of cadmium could be evidenced via the emission intensity of the interfered transition. Indeed, using the As I $234.98 \mathrm{~nm}$ transition to measure arsenic concentration, the computed intensity of the As I $228.81 \mathrm{~nm}$ line was found smaller than the measured line intensity. Thus, the cadmium concentration was adjusted so that the computed intensity of the interfered transition equals the measured one due to the contribution of the Cd I $228.80 \mathrm{~nm}$ line.

Almost all detected minor and trace elements were found in the three investigated samples, except iron that was detected for sardine only (see Fe $302 \mathrm{~nm}$ doublet). According to the criteria of line selection given in Section 4.1, the K I $404 \mathrm{~nm}$ doublet is used to deduce the amount of potassium from the analysis of the late recording. The optical thickness of the K I $769.90 \mathrm{~nm}$ transition was too large for the fraction measurement of potassium.

The elemental compositions of the three species measured via the two-step calibration-free LIBS procedure are presented Table 4 . For some minor and trace elements, mass fractions measured for sardine are reported in litterature [45, 46, 47, 48]. Except the large amount of arsenic, attributed to the industrial pollution in bay of Marseille where the seafood was caught $[27,28,29,30]$, the measured mass fractions of elements lie in the ranges of the reported mass fraction 
Table 4: Mass fractions of minor and trace elements deduced from the present LIBS analysis $C_{\text {LIBS }}$ for the three seafood species. Values reported in litterature for sardine $[45,46,47,48]$ lie in the reference ranges $\Delta C_{r e f}$.

\begin{tabular}{ccccc}
\hline & sepia & octopus & \multicolumn{2}{c}{ sardine } \\
element & $C_{\text {LIBS }}$ & $C_{\text {LIBS }}$ & $C_{\text {LIBS }}$ & $\Delta C_{\text {ref }}$ \\
\hline $\mathrm{K}(\%)$ & 1.0 & 1.7 & 1.5 & \\
$\mathrm{P}(\%)$ & 0.62 & 0.32 & 0.57 & \\
$\mathrm{Na}(\%)$ & 0.15 & 0.27 & 0.18 & \\
$\mathrm{Mg}(\mathrm{ppm})$ & 460 & 300 & 300 & \\
$\mathrm{Ca}(\mathrm{ppm})$ & 360 & 480 & 420 & \\
$\mathrm{As}(\mathrm{ppm})$ & 41 & 14 & 15 & 0.22 \\
$\mathrm{Fe}(\mathrm{ppm})$ & - & - & 16 & $13-40$ \\
$\mathrm{Si}(\mathrm{ppm})$ & 5.8 & 5.9 & 4.7 & \\
$\mathrm{Zn}(\mathrm{ppm})$ & 5.5 & 4.3 & 29 & $8-35$ \\
$\mathrm{Cu}(\mathrm{ppm})$ & 4.9 & 2.6 & 0.8 & $0.8-4.2$ \\
$\mathrm{Cd}(\mathrm{ppm})$ & 1.9 & - & - & \\
$\mathrm{B}(\mathrm{ppm})$ & 5.8 & 1.0 & 1.5 & \\
$\mathrm{Li}(\mathrm{ppm})$ & 1.3 & 1.1 & 0.29 & \\
$\mathrm{Al}(\mathrm{ppm})$ & - & 2 & 1 & \\
$\mathrm{Sr}(\mathrm{ppm})$ & 20 & 55 & 17 & \\
\hline
\end{tabular}

values.

The measurement accuracy of the two-step procedure is expected to equal the accuracy obtained with the same algorithm applied to single spectrum analysis $[25,26]$ if the required hypotheses of full and partial equilibrium are satisfied for the early and late measurements, respectively. In that case, measurement errors of a few percent may be reached if accurate spectroscopic data are available and statistical errors due to signal-to-noise limitation are negligible. The precise evaluation of the analytical performance requires further investigations with homogeneous organic samples characterized by standard techniques such as ICP-MS and ICP-AES.

\section{Conclusion}

We present a two-step procedure that enables quantitative analysis of trace elements in seafood using calibration-free laser-induced breakdown spectroscopy. The method consists of two successive measurements performed with different delays of the detector gate with respect to the laser pulse. The early recording, performed when the electron density is large enough to ensure full local thermodynamic equilibrium, serves to measure major and minor element fractions. The late recording is used to quantify trace elements, exploring the partial LTE state in which Boltzmann equilibrium distributions are limited to metal atoms. To obtain highest signal-to-noise ratio, the late recording is performed with a large gate width. Thus, to consider the variations of electron density and temperature during the time of observation, the measured spectrum is compared to the spectral radiance that takes into account the $n_{e}(t)$ - and $T(t)$ - evolutions by dividing the measurement time into two intervals, characterized by different thermodynamic states. The presented method is validated by comparing the mass fractions of some elements measured for sardine to values reported in litterature.

Beside sea food, the two-step calibration-free measurements are foreseen to be applicable to any kind of food, and more general to organic materials. Further improvements of the limits of detection and the reduction of the measurement time are achievable with a more sensitive spectroscopic apparatus.

\section{Acknowledgements}

The $\mathrm{PhD}$ thesis of C.-T. Chen was supported by the French Ministry of National Education, Research and Technology through the APREM (Alimentation des poissons planctonophages de la région marseillaise 2016-2019) programme.

[1] J. M. McLaughlin, D. R. Parker, J. M. Clarke, Metals and micronutrients - food safety issues, Field Crops Res. 60 (1999) 143-163.

[2] D. Bourn, J. J. Prescott, A comparison of the nutritional value, sensory qualities, and food safety of organically and conventionally produced foods, Crit. Rev. Food Sci. Nut. 42 (2002) 1-34.

[3] B. Sezer, G. Bilge, I. H. Boyaci, Capabilities and limitations of libs in food analysis, Trends Anal. Chem. 97 (2017) 345-353.

[4] D. W. Hahn, N. Omenetto, Laser-induced breakdown spectroscopy (LIBS), Part II: Review of instrumental and methodological approaches to material analysis and applications to different fields, Appl. Spectrosc. 66 (4) (2012) 347-419.

[5] F. J. Fortes, J. Moros, P. Lucena, L. M. Cabalin, J. J. Laserna, Laser-induced breakdown spectroscopy, Anal. Chem. 85 (2013) 640-669.

[6] L. C. Peruchi, L. C. Nunes, G. G. A. de Carvalho, M. B. B. Guerra, E. de Almeida, I. A. Rufini, D. S. Jr., F. F. Krug, Determination of inorganic nutrients in wheat flour by laser-induced breakdown spectroscopy and energy dispersive x-ray fluorescence spectrometry, Spectrochim. Acta Part B: Atom. Spectrosc. 100 (2014) 129-136.

[7] G. Bilge, B. Sezer, K. E. Eseller, H. Berberoglu, H. Koksel, I. H. Boyaci, Determination of ca addition to the wheat flour by using laser-induced breakdown spectroscopy (libs), European Food Res. Technol. 242 (2016) 1685-1692.

[8] E. C. Ferreira, E. A. Menezes, W. O. Matos, D. M. B. P. Milori, A. R. A. Nogueira, L. Martin-Neto, Determination of Ca in breakfast cereals by laser induced breakdown spectroscopy, Food Control 21 (2010) 1327-1330.

[9] B. Sezer, G. Bilge, I. H. Boyaci, Laser-induced breakdown spectroscopy based protein assay for cereal samples, J. Agricult. Food Chem. 64 (2016) 9459-9463. 
[10] V. Juvé, R. Portelli, M. Boueri, M. Baudelet, J. Yu, Spaceresolved analysis of trace elements in fresh vegetables using ultraviolet nanosecond laser-induced breakdown spectroscopy, Spectrochim. Acta Part B: Atom. Spectrosc. 63 (2008) 10471053.

[11] S. Beldjilali., W. L. Yip, J. Hermann, T. Baba-Hamed, A. Belasri, Investigation of plasmas produced by laser ablation using single and double pulses for food analysis demonstrated by probing potato skins, Anal. Bioanal. Chem. 400 (2011) 2173 2183.

[12] W. Q. Lei, J. E. Haddad, V. Motto-Ros, N. Gilon-Delepine A. Stankova, Q. L. Ma, X. S. Bai, L. J. Zheng, H. P. Zeng, J. Yu, Comparative measurements of mineral elements in milk powders with laser-induced breakdown spectroscopy and inductively coupled plasma atomic emission spectroscopy, Anal. Bioanal. Chem. 400 (2011) 3303-3313.

[13] Z. Abdel-Salam, J. A. Sharnoubi, M. A. M. A. Harith, Qualitative evaluation of maternal milk and commercial infant formulas via libs, Talanta 115 (2013) 422-426.

[14] Y. G. M. Kongbonga, H. Ghalila, M. B. Onana, Z. B. Lakhdar, Classification of vegetable oils based on their concentration of saturated fatty acids using laser induced breakdown spectroscopy (LIBS), Food Chem. 147 (2014) 327-331.

[15] G. Kim, J. Kwak, J. Choi, K. Park, Detection of Nutrient Elements and Contamination by Pesticides in Spinach and Rice Samples Using Laser-Induced Breakdown Spectroscopy (LIBS), J. Agricult. Food Chem. 60 (2012) 718-724.

[16] G. Bilge, H. M. Velioglu, B. Sezer, K. E. Eseller, I. H. Boyaci, Identification of meat species by using laser-induced breakdown spectroscopy, Meat Sci. 119 (2016) 118-122.

[17] W. Lei, V. Motto-Ros, M. Boueri, Q. Ma, D. Zhang, L. Zheng, H. Zeng, J. Yu, Time-resolved characterization of laser-induced plasma from fresh potatoes, Spectrochim. Acta Part B: Atom. Spectrosc. 64 (2009) 891-898.

[18] S. Beldjilali, D. Borivent, L. Mercadier, E. Mothe, G. Clair, J. Hermann, Evaluation of minor element concentrations in potatoes using laser-induced breakdown spectroscopy, Spectrochim. Acta Part B: Atom. Spectrosc. 65 (2010) 727 - 733.

[19] R. Agrawal, R. Kumar, S. Rai, A. K. Pathak, A. K. Rai, G. K. Rai, Libs: A quality control tool for food supplements, Food Biophys. 6 (2011) 527-533.

[20] E. Tognoni, G. Cristoforetti, S. Legnaioli, V. Palleschi, Calibration-free laser-induced breakdown spectroscopy: State of the art, Spectrochim. Acta Part B: Atom. Spectrosc. 65 (2010) $1-14$.

[21] T. Takahashi, B. Thornton, Quantitative methods for compensation of matrix effects and self-absorption in laser induced break down spectroscopy signals of solids, Spectrochim. Acta Part B: Atom. Spectrosc. 138 (2017) 31-42.

[22] H. R. Griem, Plasma spectroscopy, Academic, New York, 1964.

[23] A. D. Giacomo, R. Gaudiuso, M. Dell'Aglio, A. Santagata, The role of continuum radiation in laser induced plasma spectroscopy, Spectrochim. Acta Part B: Atom. Spectrosc. 65 (2010) 385-394.

[24] J. Hermann, D. Grojo, E. Axente, V. Craciun, Local thermodynamic equilibrium in a laser-induced plasma evidenced by blackbody radiation, Spectrochim. Acta Part B: Atom. Spectrosc. 144 (2018) 82-86.

[25] C. Gerhard, J. Hermann, L. Mercadier, L. Loewenthal, E. Axente, C. R. Luculescu, T. Sarnet, M. Sentis, W. Viöl, Quantitative analyses of glass via laser-induced breakdown spectroscopy in argon, Spectrochim. Acta Part B: Atom. Spectrosc. 101 (2014) 32-45.

[26] J. Hermann, A. Lorusso, A. Perrone, F. Strafella, C. Dutouquet, B. Torralba, Simulation of emission spectra from nonuniform reactive laser-induced plasmas, Phys. Rev. E 92 (2015) 053103 $1-15$.

[27] D. Faget, Marseille et la mer : Hommes et environnement marin (XVIIIe-XXe siècle), Presses universitaires, Rennes, 2011.

[28] B. Oursel, C. Garnier, G. Durrieu, S. Mounier, D. Omanovic, Y. Lucas, Dynamics and fates of trace metals chronically. input in a Mediterranean coastal zone impacted by a large urban area, Mar. Poll. Bull. 69 (2013) 137-149.

[29] D. Banaru, F. Carlotti, A. Barani, G. Gregori, N. Neffati, M. Harmelin-Vivien, Seasonal variation of stable isotope ratios of size-fractionated zooplankton in the Bay of Marseille (NW Mediterranean Sea), J. Plankton Res. 36 (2014) 145-156.

[30] M. Ourgaud, S. Ruitton, H. Bourgogne, P. Bustamante, C. Churlaud, G. Guillou, B. Lebreton, M. L. Harmelin-Vivien, Trace elements in a Mediterranean scorpaenid fish: Bioaccumulation processes and spatial variations, Prog. Oceanogr. 163 (2018) 184-195.

[31] B. Millet, C. Pinazo, D. Banaru, R. Pages, P. Guiart, I. Pairaud, Unexpected spatial impact of treatment plant discharges induced by episodic hydrodynamic events: Modelling Lagrangian transport of fine particles by Northern Current intrusions in the bays of Marseille (France), PLoS ONE 13 (2018) e0195257 1-25.

[32] J. Hermann, D. Grojo, E. Axente, C. Gerhard, M. Burger, V. Craciun, Ideal radiation source for plasma spectroscopy generated by laser ablation, Phys. Rev. E 96 (2017) 053210 1-6.

[33] M. Dell'Aglio, M. Lopez-Claros, J. J. Laserna, S. Longo, A. De Giacomo, Stand-off laser induced breakdown spectroscopy on meteorites: calibration-free approach, Spectrochim. Acta Part B: Atom. Spectrosc. 147 (2018) 87-92.

[34] J. Cooper, Plasma spectroscopy, Rep. Prog. Phys. 29 (1966) 35130

[35] X. Z. Zhao, L. J. Shen, T. X. Lu, K. Niemax, Spatial distributions of electron-density in microplasmas produced by laser ablation of solids, Appl. Phys. B: Photophys. Laser Chem. 55 (1992) 327-330.

[36] E. Tognoni, V. Palleschi, M. Corsi, G. Cristoforetti, N. Omenetto, I. Gornushkin, B. W. Smith, J. D. Winefordner, From sample to signal in laser-induced breakdown spectroscopy: a complex route to quantitative analysis, in: A. W. Miziolek, V. Palleschi, I. Schechter (Eds.), Laser-induced breakdown spectroscopy, Cambridge University, Berlin, 2006, pp. 122-194.

[37] M. Burger, J. Hermann, Stark broadening measurements in plasmas produced by laser ablation of hydrogen containing compounds, Spectrochim. Acta Part B: Atom. Spectrosc. 122 (2016) 118-126.

[38] J. Hermann, C. Dutouquet, Local thermal equilibrium plasma modeling for analyses of gas-phase reactions during reactivelaser ablation, J. Appl. Phys. 91 (2002) 10188-10193.

[39] Y. B. Zel'dovich, Y. P. Raizer, Physics of Shock Waves and High Temperature Phenomena, Academic, New York, 1966.

[40] J. Hermann, E. Axente, V. Craciun, A. Taleb, F. Pelascini, Evaluation of pressure in a plasma produced by laser ablation of steel, Spectrochim. Acta Part B: Atom. Spectrosc. 143 (2018) 63-67.

[41] A. A. Radzig, B. M. Smirnov, Reference data on atoms, molecules and ions, Springer, Berlin, 1985.

[42] A. D. Giacomo, J. Hermann, Laser-induced plasma emission: from atomic to molecular spectra, J. Phys. D: Appl. Phys. 50 (2017) 183002 1-17.

[43] M. A. Gigosos, M. A. Gonzalez, V. Cardenoso, Computer simulated Balmer-alpha, -beta, and -gamma Stark line profiles for non-equilibrium plasma diagnostics, Spectrochim. Acta Part B: Atom. Spectrosc. 58 (2003) 1489-1504.

[44] A. Kramida, Y. Ralchenko, J. Reader, NIST Atomic Spectra 
Database (version 5.5.6), National Institute of Standards and Technology, Gaithersburg, MD (2018).

URL http://physics.nist.gov/asd

[45] M. Canli, G. Atli, The relationships between heavy metal (Cd, $\mathrm{Cr}, \mathrm{Cu}, \mathrm{Fe}, \mathrm{Pb}, \mathrm{Zn}$ ) levels and the size of six mediterranean fish species, Environ. Poll. 121 (2003) 129-136.

[46] A. Ikem, N. O. Egiebor, Assessment of trace elements in canned fishes (mackerel, tuna, salmon, sardines and herrings) marketed in Georgia and Alabama (United States of America), J. Food Comp. Anal. 18 (2005) 771-787.

[47] M. Tuzen, M. Soylak, Determination of trace metals in canned fish marketed in turkey, Food Chem. 101 (2007) 1378-1382.

[48] S. Mol, Levels of heavy metals in canned bonito, sardines, and mackerel produced in turkey, Bio. Trace Elem. Res. 143 (2011) 974-982. 